\title{
REVIEW
}

\section{Pseudo-templated transcription in prokaryotic and eukaryotic organisms}

\author{
Jean-Philippe Jacques and Daniel Kolakofsky \\ Department of Microbiology, University of Geneva Medical School, CH-1211 Geneva, Switzerland
}

\section{What is pseudo-templated transcription?}

Pseudo-templated transcription describes a process that results in the production of a homopolymeric stretch of RNA directed by a shorter stretch (usually 3-7) of complementary nucleotides on the template (either RNA or DNA). The effects of pseudo-templated transcription are very diverse. In some cases, strictly homopolymeric RNAs are made; in others, pseudo-templated nucleotides are added at either the $5^{\prime}$ or $3^{\prime}$ ends of otherwise normally templated transcripts; and finally, sometimes pseudo-templated nucleotides are inserted at a specific internal site during elongation within an otherwise normally templated RNA. As a result, pseudo-templated transcription is involved in events such as transcriptional frameshifting (programmed or not) and synthesis of the poly(A) head or poly(A) tail of various mRNAs. We will try to describe the general features of pseudo-templated transcription and assess its importance within a more general framework.

Reiterative copying of a template was first described by Chamberlin and Berg (1964, and references therein). They observed production of polyadenylic acid in an in vitro system where denatured natural DNA or oligo $(T)$ is provided as a template and ATP, the sole substrate for the Escherichia coli RNA polymerase holoenzyme (RNAP). With denatured DNA, production of poly(A) was inhibited by low concentration of the other three NTPs and was proposed to be directed by a stretch of Ts on the DNA template strand. With oligo(T) as template, the resulting poly(A) exceeded the length of the template by as much as 20 -fold. Similar examples were reported at this time, suggesting that under certain conditions RNAP had the ability to direct slippage during RNA synthesis (for review, see Yager and Von Hippel 1987, and references therein).

\section{Pseudo-templated transcription in prokaryotes}

\section{Production of transcripts with heterogeneous $5^{\prime}$ ends}

As early as 1975, Schaller and co-workers (Heyden et al. 1975; Nüsslein and Schaller 1975), while investigating bacteriophage fd promoters, noticed some discrepancy between the sequence of gene $X$ and the $5^{\prime}$ end of its transcripts (Table 1). Upon fingerprinting the RNAs produced in vitro, they found the $5^{\prime}$ ends to be
$5^{\prime}-(\mathrm{G})_{n}$ UAAG. . , with $n$ ranging from 1 to 4 . Since there are only three Gs near the transcription startpoint on the DNA-coding strand, a variable number of Gs at the 5' end of the transcripts were said to indicate a certain "sloppiness" of the polymerase during initiation. Furthermore, oligo(G) were produced with GTP as the only substrate, and oligo $(G)$ production was significantly reduced when UTP was added. As UTP is next incorporated after GTP in the RNA, UTP seemed to prevent the RNAP from slipping reiteratively and predisposed it to a strictly templated mode of transcription.

It is possible that similar observations were under-reported for the next 10 years because they were considered to be experimental artifacts. However, in 1984, while investigating the insertion sequence IS1, Machida et al. examined the transcripts produced in vitro from the ins $A$ promoter. The ins $A$ transcripts were heterogeneous in size, and RNA sequencing revealed variations at the $5^{\prime}$ end of both abortive and full-length products

Table 1. Pseudo-templated transcription by E. coli RNA polymerase

\begin{tabular}{|c|c|c|}
\hline GENE & TEMPLATE & PRODUCT \\
\hline $\begin{array}{l}1 / \text { Slippery Promoters } \\
x \text { (phage fd) }\end{array}$ & 3. CCCATT & $(G)_{n}$ UAA. . . \\
\hline insA (IS1) & 3' AAAAAC & (U) ${ }_{1-8}$ UUUUG... \\
\hline mutant tet (pBR322) & 3. TTITAA & (A) ${ }_{1-7}$ AAAUU $\ldots$ \\
\hline$P_{R}^{\prime} \quad$ (phage 82) & $3^{\prime}$ TTTAGA & (A) $\underset{1-18}{\text { AAUCU } \ldots}$ \\
\hline mutant sar (phage P22) & 3' $\mathrm{AAAACC}$ & $\operatorname{poly}(U)$ \\
\hline $\begin{array}{l}\text { 2/ Internal slippage si } \\
\text { mutant lacZ }\end{array}$ & $\begin{array}{l}\frac{(\mathrm{A})}{10-11} \\
(\mathrm{~T})_{10-11}\end{array}$ & $\begin{array}{l}\cdots(U)_{n>11} \\
\cdots(A)_{n>11}\end{array}$ \\
\hline
\end{tabular}

For each gene or promoter, template strand and RNA products are indicated. Slippery sites on the template are underlined. Note that the template is given $3^{\prime}$ to $5^{\prime}$ and the product $5^{\prime}$ to $3^{\prime}$. Dots at one end of the product indicate that the RNA is longer on this end. 
that were $5^{\prime}-(\mathrm{U})_{n}$ GAGG $\ldots$, with $n$ ranging from 5 to 12. The presence of five Ts on the coding strand at the startpoint region was proposed to explain such heterogeneity: "Stuttering" of the RNAP would induce incorporation of one to seven pseudo-templated Us before the polymerase assumes templated transcription.

Other examples have since been reported where RNAP stuttering occurs during initiation of transcription, even in the presence of all four NTPs. These involve production of an oligo(A) stretch: (1) at the 5' end of a late phage T4 transcript by RNAP holoenzyme containing the late gene-specific $\sigma$ subunit (Kassavetis et al. 1986); (2) at the $5^{\prime}$ end of pBR322 tet transcripts from various mutant promoters (Harley et al. 1988); and (3) at the $5^{\prime}$ end of the $P_{\mathrm{R}}{ }^{\prime}$ (late operon) transcripts of bacteriophage 82 /Guo and Roberts 1990).

In the case of the mutant tet promoters, Harley et al. $(1988,1990)$ reported that an RNA "ladder" of poly(A) was produced when only ATP was present. Comparable results were reported by Martin et al. (1988) for T7 RNA polymerase, a small $(100 \mathrm{kD})$ single-subunit polymerase known for its high processivity. A homopolymeric poly $(G)$ ladder was produced when GTP was the only substrate provided (curiously, the ladder stopped abruptly at the 14-mer). When the assay was performed with all four NTPs, however, no stuttering products were observed, and initiation of transcription was rigorously templated.

All of the results reported with the various bacterial promoters were obtained in vitro. There is no direct indication whether they also occur in vivo. However, comparable results to that obtained with $E$. coli RNAP at slippery promoters were observed with polyomavirus upon characterization of the early region transcripts (see below and Table 2). During initiation of transcription, both in infected cells and in a cell-free system, the viral polymerase has a propensity to slip backward a few times before switching to a templated mode of transcription (Cowie et al. 1982).

Table 2. Pseudo-templated transcription by DNA virus RNA polymerases

\begin{tabular}{|ccc|}
\hline GENE & TEMPLATE & PRODUCT \\
\hline $\begin{array}{c}\text { * papovavirus } \\
\text { (e.g., Polyoma) } \\
\text { early transcripts }\end{array}$ & $3^{\prime}$ TAAAGTC & $\begin{array}{c}\text { AUU (U) }{ }_{1-2} \text { CAG... } \\
\text { (AUUU) }{ }_{1-2} \text { CAG... }\end{array}$ \\
$\begin{array}{c}\text { poxvirus } \\
\text { (e.g., Vaccinia) } \\
11 K \text { \& other late transcripts }\end{array}$ & 3' ATTTAC & poly(A) head \\
\hline
\end{tabular}

For each gene or promoter, template strand and RNA products are indicated. Slippery sites on the template are underlined. Note that the template is given $3^{\prime}$ to $5^{\prime}$ and the product $5^{\prime}$ to $3^{\prime}$. Dots at one end of the product indicate that the RNA is longer on this end.

\section{Production of polyuridylic acid from $P_{\text {sar }}$}

The sar gene encodes a small antisense RNA involved in the immunity circuitry of the Salmonella phage P22 (Liao et al. 1987; Wu et al. 1987). A point mutation in $P_{\text {sar }}$ (a G $\rightarrow \mathrm{T}$ transversion at the startpoint) creates a stretch of four As on the template strand from -1 to +3 . This stretch directs pseudo-templated synthesis of poly $(\mathrm{U})$ "transcripts" in the presence of all four NTPs under standard in vitro conditions (Jacques and Susskind 1990). As opposed to the previous cases, where the polymerase stutters before ultimately escaping to the elongation mode and completing templated transcription, in the sar $+1 T$ mutant the RNAP only idles on the promoter. Many poly(U) molecules of variable lengths (as long as 150 -mers) are released by the same "idling complex," in a monotonic fashion with a constant probability of slippage after each UMP has been added for transcripts longer than 5-mers. A model that would explain the mechanism is represented in Figure 1. Sequence requirements for efficient transcription from prokaryotic promoters are well known, and many point mutations at or near the various consensus regions (e.g., the -35 and -10 hexamers/ are known for their effect on the formation of open polymerase-promoter complexes. The $s a r+1 T$ mutation has an unprecedented effect on gene expression; it severely reduces production of templated sar transcripts, both in vitro and in vivo, and directs production of poly(U).

\section{Transcriptional frameshifting}

All of the above examples of pseudo-templated transcription involve slippage during initiation, and, except for sar, they have little or no consequences on the transmission of relevant genetic information. However, pseudotemplated transcription can also occur during elongation with E. coli RNAP. Using an artificial template that featured an internal stretch of 11 As or Ts, Wagner et al. (1990) observed that in vivo, E. coli RNAP (but not Saccharomyces cerevisiae polymerase II) loses track of the number of As or Us incorporated into the elongating mRNA. As a result, there is transcriptional frameshifting, and the proteins produced come out of this track translated in all three frames. Wagner et al. pointed out that a stretch of 11 As or Ts has not as yet been found in $E$. coli-coding sequences, presumably because they might lead to frameshifting.

\section{Heads and tails of eukaryotic virus mRNAs}

Vaccinia, a member of the poxvirus family, is a DNA virus that replicates in the cytoplasm of the host cell, using its own transcriptional machinery (for review, see Moss 1990). Transcription initiation of most of the late genes is directed by the highly conserved 3'-TAAATG motif on the coding strand (Table 2). Within this sequence are both the transcription (one of the internal As) and the translation (ATG) start sites (Weir and Moss 1984; Bertholet et al. 1985; Hänggi et al. 1986). The com- 


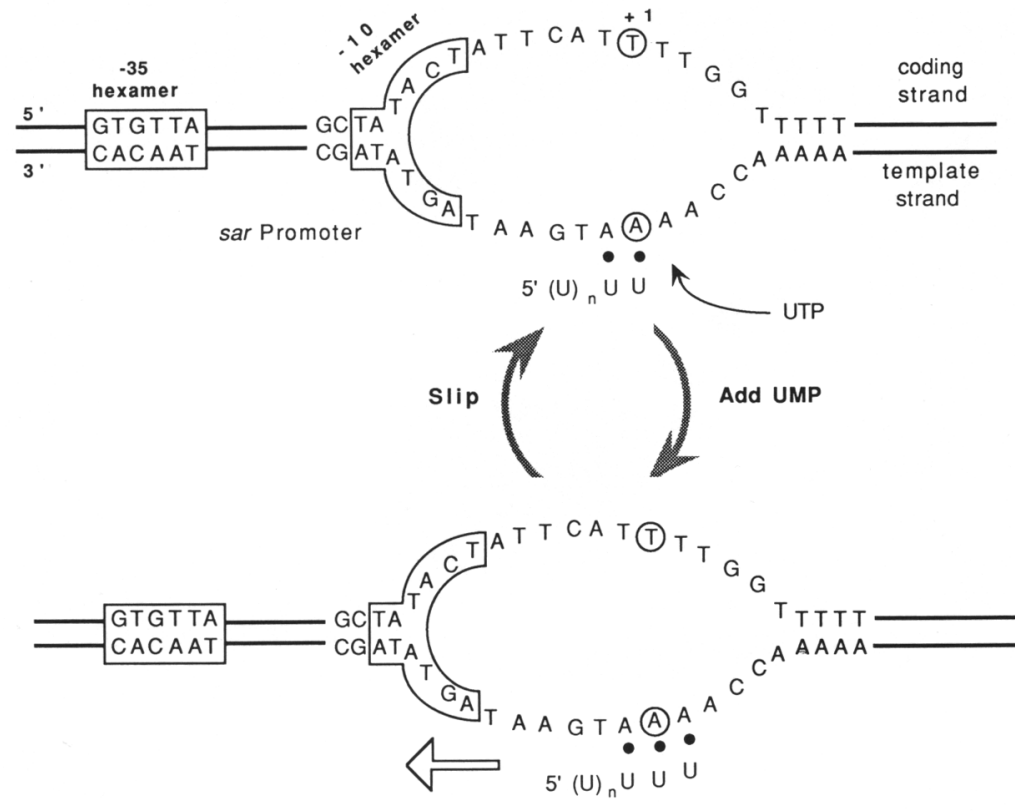

Figure 1. A model for pseudo-templated transcription from the $s a r+1 T$ promoter. The relevant DNA sequence at the promoter is shown. The startpoint for transcription is circled, and the -10 and -35 hexamers are boxed. Dots indicate hydrogen bonds between the DNA template and the RNA. plement of the three As within the motif (i.e., the three Ts on the template strand) has been proposed to be the pseudo-template for synthesis of a poly(A) head, which immediately follows the capped $5^{\prime}$ end of the late transcripts (Bertholet et al. 1986, 1987; Patel and Pickup 1987; Schwer et al. 1987; Schwer and Stunnenberg 1988). Results from various in vitro systems (Schwer and Stunnenberg 1988; Wright and Moss 1989) support the pseudo-templated polymerization hypothesis for the poly(A) head. Remarkably, the length of the poly(A) head, $\sim 35 \mathrm{As}$, is rather constant both in vitro and in vivo. The significance of this head is not established, but it might be essential for translation, as there are few examples of eukaryotic mRNAs with functional ATGs so close to their $5^{\prime}$ ends. It may also enhance the translational efficiency of the late transcripts either directly or by increasing their stability.

Not only is pseudo-templated transcription involved in formation of a poly(A) head, but it is also used to form the poly(A) tails of some viral transcripts. Rhabdoviruses such as vesicular stomatitis virus (VSV) and Paramyxoviruses such as Sendai and mumps virus have nonsegmented RNA genomes of negative polarity (i.e., complementary to the viral mRNAs) (for review, see Banerjee 1987; Kolakofsky and Roux 1987). Orthomyxoviruses (influenza virus) are also negative-strand viruses but contain eight genome segments. The polymerase /transcriptase) responsible for mRNA synthesis is virus encoded and is present in the virus particles. Because animal cells do not contain RNA-dependent RNA polymerases, it would not be possible to start the replicative cycle without the viral polymerase in the virions. Purified virions can therefore also carry out mRNA synthesis, and the mRNAs made in vitro are both capped and polyadenylated.

When the 3 ' ends of the viral mRNAs were mapped on the viral genome, the first five to eight As of the poly(A) tail were found to be complementary to five to eight Us present at the end of each cistron (Table 3). However, because the poly $(\mathrm{A})$ tail is normally 100 or more residues in length, the synthesis of the remainder of the tail was suggested to be due to polymerase stuttering on the Urun of the template (McGeoch 1979; Rose 1980; Schubert et al. 1980; Robertson et al. 1981; Giorgi et al. 1983; Gupta and Kingsbury 1984). Mutants of VSV exist in which abnormally long (>1000-nucleotide) poly(A) tails are formed, and reconstitution studies have shown that this property is associated with the $\mathrm{L}$ protein, one of the polymerase components (Hunt et al. 1984). Curiously, when mRNAs are made in vitro with some strains of VSV in the presence of $S$-adenosyl-homocysteine (an inhibitor of cap methylation), the poly(A) tail grows to

Table 3 Pseudo-templated transcription by (-)RNA virus transcriptases

\begin{tabular}{|c|c|c|}
\hline GENE & TEMPLATE & PRODUCT \\
\hline \multicolumn{3}{|l|}{$\begin{array}{l}\text { "rhabdovirus } \\
\text { (e.g., VSV) }\end{array}$} \\
\hline $\begin{array}{l}\text { viral transcripts } \\
\text { * orthomyxovirus } \\
\text { (e.g., Influenza) }\end{array}$ & $3^{\prime} \mathrm{AC}(\underline{\mathrm{U}}), \mathrm{GA}$ & poly(A) tail \\
\hline viral transcripts & $\underline{(U)}_{5-7}$ & $\operatorname{poly}(\mathrm{A})$ tail \\
\hline \multicolumn{3}{|l|}{$\begin{array}{l}\text { paramyxovirus } \\
\text { (e.g., Parainfluenza) }\end{array}$} \\
\hline viral transcripts & $(\underline{U})_{6-8}$ & poly(A) tail \\
\hline$P$ & $3^{\prime}$ UUYUCCC & $\operatorname{AARAGGG}(\mathrm{G})_{1-2} \ldots$ \\
\hline
\end{tabular}

$\mathrm{Y}=\mathrm{C}$ or $\mathrm{U}_{;} \mathrm{R}=\mathrm{A}$ or $\mathrm{G}$. 
1000 or more residues in length (Rose et al. 1988). This suggests that there may be a link between $5^{\prime}$ - and $3^{\prime}$-end formation of the sequential mRNAs. In support of this notion, Iverson and Rose (1981) have shown that the VSV polymerase pauses at the gene junction during mRNA synthesis.

\section{Programmed stuttering within the coding region of Paramyxovirus $P$ genes: a form of $m R N A$ editing}

Paramyxovirus $P$ genes contain an overlapping open reading frame $|O R F|$ that begins near the middle of the gene, and separate mRNAs are produced to express the alternate downstream ORF (Fig. 2). One mRNA is an exact copy of the gene, whereas the other(s) contain a small number of Gs inserted at a specific site, within a short run of Gs. These insertions fuse the alternate downstream ORF to the common amino-terminal sequences (Fig. 2). Since pseudo-templated polymerization was suggested previously as the mechanism for poly(A) tail formation on these mRNAs, a similar slippage mechanism, but of a more precise nature, was suggested for the G insertions (Thomas et al. 1988; Cattaneo et al. 1989).

Work with Sendai virus supports this mechanism (Vidal et al. 1990a). mRNAs made in vitro with purified virions contain the same precise insertions as those made in vivo, suggesting that the insertion activity is virus encoded. Moreover, in cells coinfected with Sendai virus and a vaccinia virus recombinant expressing the Sendai virus $P$ gene, the natural $P$ mRNA contains the inserted Gs as usual, whereas the mRNA made from the vaccinia DNA is not modified. The inability of the insertion activity to act in trans is consistent with the idea that the insertions take place cotranscriptionally and only during synthesis by the Sendai polymerase on the Sendai template.

The G-insertion mechanism of Paramyxovirus, which is a form of mRNA editing, is remarkable in several ways. (1) Unlike poly(A) tail formation, the mechanism is designed to occur only some of the time, and the ratio of inserted to uninserted mRNA is tightly controlled (Vidal et al. 1990a). (2) The mechanism is adaptable to the particular coding strategies of different viral $P$ genes. In the mumps group, the uninserted mRNA codes for the $\mathrm{V}$ protein, whereas the mRNA that codes for $\mathrm{P}$ requires an insertion of two Gs (Paterson and Lamb 1990). In the Sendai group, the uninserted mRNA codes for the $P$ protein, and an mRNA with a single $G$ insertion codes for the $\mathrm{V}$ protein. In the latter group, $>80 \%$ of the mRNAs with insertions have added only a single $G$, whereas in the former group, the insertion of two Gs is the predominant event and mRNAs with only $+1 G$ rarely, if ever, occur. (3) Bovine parainfluenza virus 3 (bPIV3) contains two alternate reading frames downstream (called $\mathrm{V}$ and D) (Fig. 2), which are accessed from a single mRNA insertion site (a variation of the Sendai coding strategy). Here, insertions occur twice as frequently as in Sendai virus, and one to six Gs are added with almost equal frequency (Pelet et al. 1991). The result is that half the mRNAs code for $\mathrm{P}$, and the other half code equally for $\mathrm{V}$ and $\mathrm{D}$ proteins.

$1 /$ Mumps, Simian Virus 5

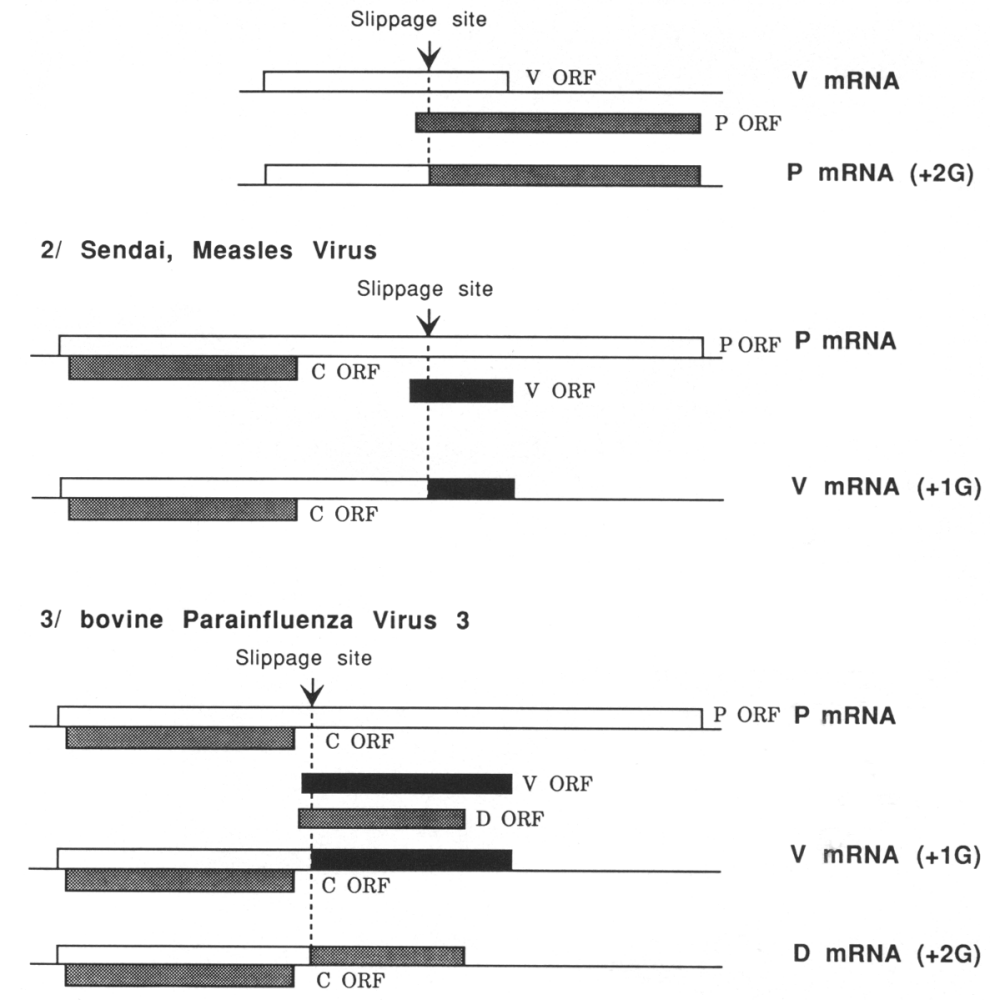

2/ Sendai, Measles Virus

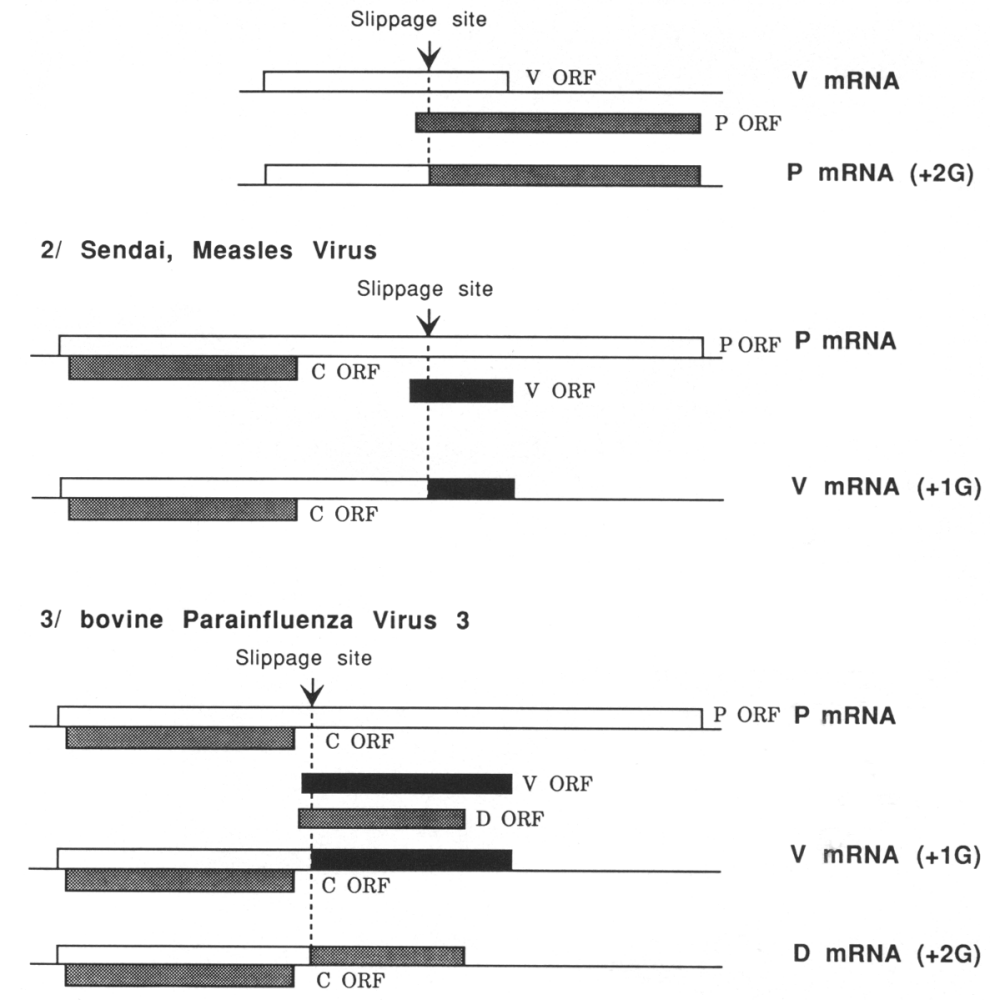

3/ bovine Parainfluenza Virus 3

Slippage site

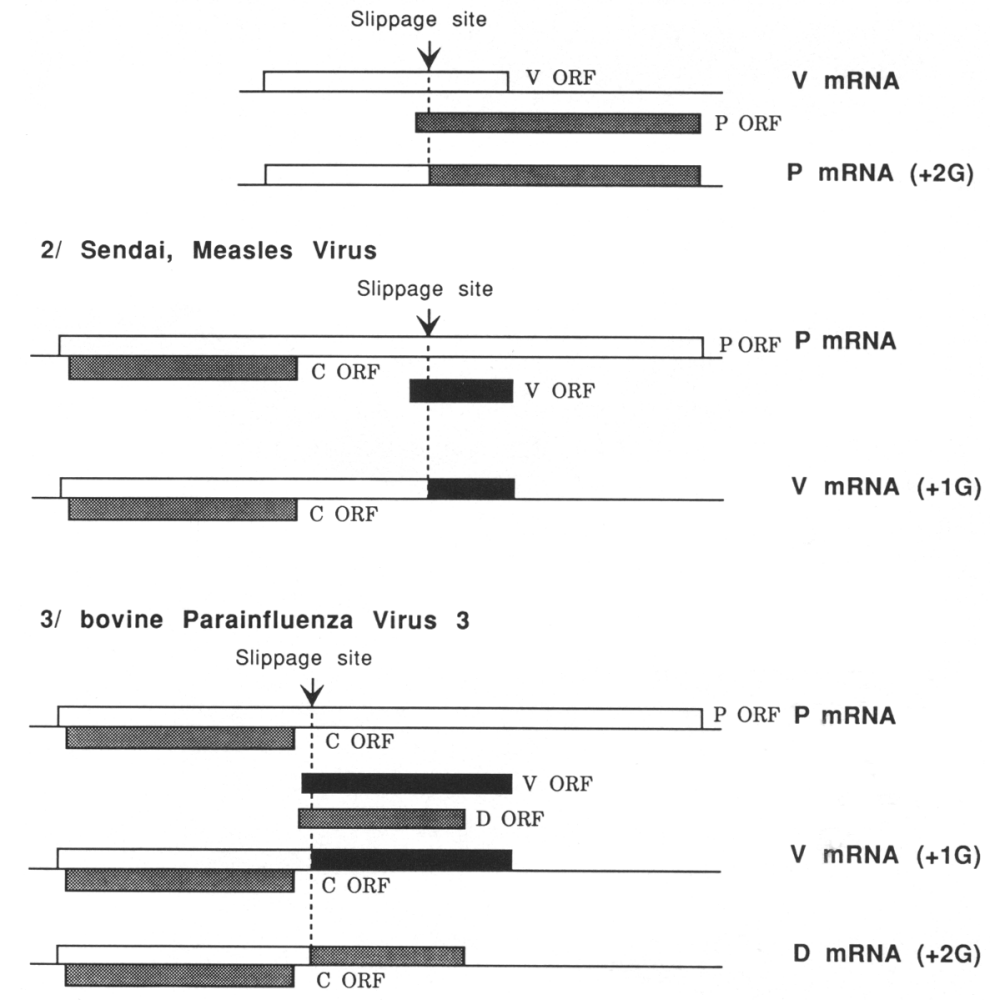

Figure 2. Schematic representation of Paramyxovinus $P$ gene and mRNAs. For each group, the upper line shows the mRNA which is an exact copy of the gene. The three possible ORFs are indicated by open, hatched, and black boxes. Below are shown the various overlapping ORFs that would be fused to the amino-terminal segment upon $\mathrm{G}$ insertion. The C ORFs, when present, are accessed by alternate initiation codons. 
A stuttering model to explain how this mechanism can be adapted to serve the various $P$-gene-coding strategies has been suggested (Fig. 3) (Vidal et al. 1990b; Pelet et al. 1991). The polymerase is proposed to pause on the insertion sequence, which is always $3^{\prime}$-UUYUCCC fon the template strand) (Table 3). In some cases, the pause is insufficient for slippage to occur, and uninserted mRNAs are made. When the pause is long enough, slippage of the 3' end of the nascent mRNA relative to the template occurs, which leads to the insertions. The distribution of polymerase pause lengths at a given site then determines the fraction of mRNAs with insertions. For the Sendai group (whose site is 3'-UUUUCCC), a 1-base slippage is preferred as this is the most stable misalignment intermediate. For the mumps group $\left[3^{\prime}\right.$ UUCUCCC), a 1-base slippage creates an unfavorable A : $\bar{C}$ base pair, and so a 2-base slippage is the preferred event.

Of course, once the first pseudo-templated G or Gs have been added, the polymerase is back to the pause site. It should now be easier to slip again because of the G : U base pairs created, yet only one round of slippage/ insertion occurs most of the time. To account for this, it is proposed that the pause has been eliminated during the first round of slippage/insertion for the above viruses. For bPIV3, the pause would not be eliminated, leading to the very broad distribution of inserted Gs.

\section{Key elements of pseudo-templated transcription and related mechanisms}

A priori, two elements can be proposed for pseudo-templated transcription to operate: first, a slippage site, which is a homopolymeric run on the DNA or RNA template, and which can be as short as 3 nucleotides; second, a pause-inducing element that would allow positioning of the polymerase at the slippage site. This element is apparently not needed for slippage of $E$. coli RNAP during elongation at a run of 11 As or Ts (Wagner et al. 1990). The pause-inducing element can be a promoter, at which the polymerase idles before initiating templated transcription. For synthesis of the poly(A) tail of negative-strand RNA virus mRNAs, this element would be part of the termination signal. For influenza virus, the polyadenylation site abuts the panhandle formed by the complementary ends of each genome segment, and the panhandle structure has been proposed as a key element of this mechanism (Robertson et al. 1981;

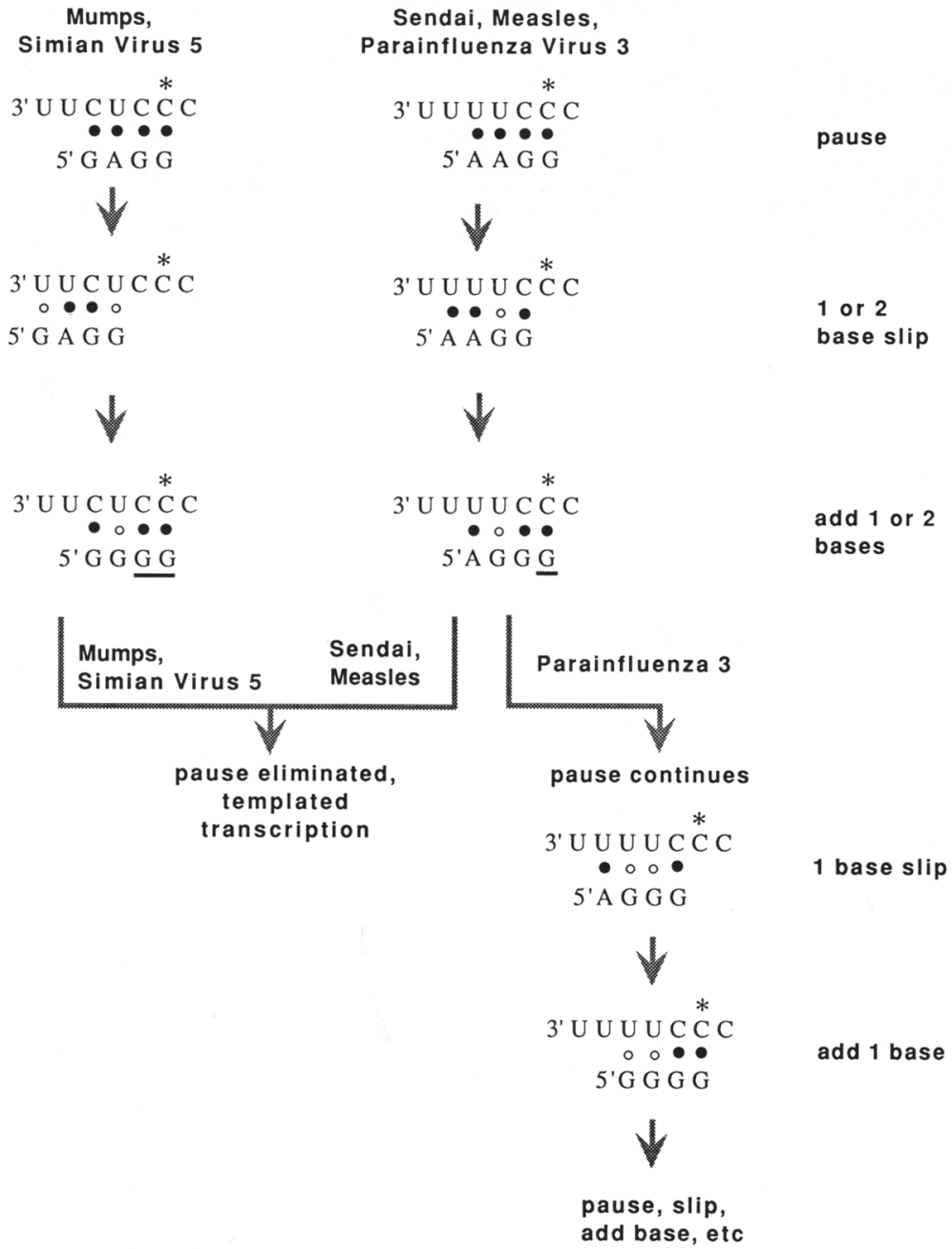

Figure 3. A model to explain programmed pseudotemplated transcription for Paramyxovirus $P$ genes. The polymerase pauses after adding the second $G$ to the mRNA, and the mRNA slips 2 bases (for mumps group) or 1 base upstream (Sendai group), creating 2 or $1 \mathrm{G}$ : U base pairs, respectively $(0)$ Addition of the pseudo-templated Gs brings the polymerase back to the pause site (indicated by an asterisk above the template stand), creating a two-G or one-G insertion (underlined). For all but bPIV3, the pause is eliminated and the polymerase resumes templated transcription. For bPIV3, the pause is not eliminated, allowing multiple rounds of slippage and insertion. In this model, only $4 \mathrm{bp}$ between the nascent mRNA and the template are shown. 
Hsu et al. 1987). In the case of the Paramyxovirus $P$ gene, where pseudo-templated transcription occurs in the middle of the transcripts, the reasons why the polymerase would pause on the slippery sequence are unclear. Strong secondary structures around the editing site can be predicted for SV5 and mumps virus (Thomas et al. 1988; Takeuchi et al. 1990) but not for the other viruses, and there is no indication as yet that these templates /unlike influenza virus) can form secondary structures. Polymerase pausing during editing of the $P$ mRNA thus remains to be shown. This putative mechanism is reminiscent of ribosomal frameshifting induced by formation of a stemloop (Jacks et al. 1988) or a pseudoknot (Brierley et al. 1989).

A variation on the pseudo-templated theme is that of telomere polymerization (for review, see Blackburn 1984; Boeke 1990). The various telomere terminal transferases (telomerases) are involved in reiterative addition of telomeric repeats such as (TTGGGG) ${ }_{n}$ in tetrahymena or (TTGGGA) $\left.\right|_{n}$ in human. The telomerase is a ribonucleoprotein complex that includes the RNA template used in an inchworm mechanism for telomere polymerization (Shippen-Lentz and Blackburn 1990; Yu et al. 1990). Telomere addition is an example of pseudo-templated reverse transcription. Finally, pseudo-templated transcription is reminiscent of proposed mechanisms for generation of frameshift and other types of mutations by misalignment of the template and nascent DNA strands during replication (Kunkel and Soni 1988).

\section{Conclusions and prospects}

There are various cases where the transcript is not a direct replica of the coding DNA (or RNA) strand. Several mechanisms termed "RNA editing" have been described (for review, see Simpson and Shaw 1989; Benne 1990). Pseudo-templated transcription as we have defined it here is one of them; it implies a localized discrepancy between a template and the corresponding mRNA, and this discrepancy is introduced during the primary transcription.

Several key features can be proposed that characterize pseudo-templated transcription. The variety of organisms and polymerases potentially or actually involved is quite large as well as the expected biological consequences. Pseudo-templated transcription sometimes affects promoter clearance, or is involved in the addition of a poly $(A)$ head or a poly $(A)$ tail to a transcript, and can also be involved in transcriptional frameshifting. The requirements in terms of primary sequence are not well understood, nor is the commitment for the various polymerases to "switch" from one mode of transcription (e.g., templated) to the other (pseudo-templated). One wonders how widely pseudo-templated transcription is distributed. As opposed to slippage during replication, pseudo-templated transcription is not expected to have much impact on the transmission of the genetic information. However, in the case of human respiratory syncytial virus (a more distant member of the Paramyxovir- idae), pseudo-templated synthesis takes place during genome replication and plays an important role in the mechanism whereby the virus mutates to escape neutralization by antibodies (Garcia-Barreno et al. 1990). Although pseudo-templated transcription is hardly noticeable in some cases, and difficult to prove in others, the number of examples is steadily increasing.

\section{Acknowledgments}

We are grateful to M.M. Susskind for helpful discussions and for supporting J.-P.J. while a graduate student in her laboratory. This work was supported by grant GM36811 from the National Institutes of Health to M.M. Susskind, by an EMBO postdoctoral fellowship to J.-P.J., and by a grant from the Swiss National Science Foundation to D.K.

\section{References}

Banerjee, A.K. 1987. Transcription and replication of rhabdoviruses. Microbiol. Rev. 51: 66-87.

Benne, R. 1990. RNA editing in trypanosomes: Is there a message? Trends Genet. 6: 177-181.

Bertholet, C., R. Drillieu, and R. Wittek. 1985. One hundred base pairs of $5^{\prime}$ flanking sequence of a vaccinia virus late gene are sufficient to temporally regulate late transcription. Proc. Natl. Acad. Sci. 82: 2096-2100.

Bertholet, C., P. Stocco, E. Van Meir, and R. Wittek. 1986. Functional analysis of the $5^{\prime}$ flanking sequence of a Vaccinia virus late gene. $E M B O$ \%. 5: 1951-1957.

Bertholet, C., E. Van Meir, B. Heggeler-Bordier, and R. Wittek. 1987. Vaccinia virus produces late mRNAs by discontinuous synthesis. Cell 50: 153-162.

Blackburn, E.H. 1984. Telomeres: Do the end justify the means? Cell 37: 7-8.

Boeke, J.D. 1990. Reverse-transcriptase, the end of the chromosome, and the end of life. Cell 61: 193-195.

Brierley, I., P. Digard, and S.C. Inglis. 1989. Characterization of an efficient Coronavirus ribosomal frameshifting signal: Requirement for an RNA pseudoknot. Cell 57: 537-547.

Cattaneo, R., K. Kaelin, K. Baczko, and M.A. Billeter. 1989. Measles virus editing provides an additional cysteine-rich protein. Cell 56: 759-764.

Chamberlin, M. and P. Berg. 1964. Mechanism of RNA polymerase action: Characterization of the DNA-dependent synthesis of polyadenylic acid. I. Mol. Biol. 8: 708-726.

Cowie, A., P. Jat, and R. Kamen. 1982. Determination of sequences at the capped $5^{\prime}$ ends of polyoma virus early region transcripts synthesized in vivo and in vitro demonstrates an unusual microheterogeneity. J. Mol. Biol. 159: 225-255.

Garcia-Barreno, B., A. Portela, T. Delgado, J.A. López, and J.A. Melero. 1990. Frameshift mutations as a novel mechanism for the generation of neutralization resistant mutants of human respiratory syncitial virus. EMBO I. 9: 4181-4187.

Giorgi, C., B.M. Blumberg, and D. Kolakofsky. 1983. Sendai virus contains overlapping genes expressed from a single mRNA. Cell 35: 829-836.

Guo, H.-C. and J.W. Roberts. 1990. Heterogeneous initiation due to slippage at the bacteriophage 82 late gene promoter in vitro. Biochemistry 29: 10701-10709.

Gupta, M. and D.W. Kingsbury. 1984. Complete sequences of the intergenic and mRNA start signals in the Sendai virus 
genome: Homologies with the genome of Vesicular Stomatitis virus. Nucleic Acids Res. 12: 3829-3841.

Hänggi, M., W. Bannwarth, and H.G. Stunnenberg. 1986. Conserved TAAAAT motif in vaccinia virus late promoters: Overlapping TATA box and site of transcription initiation. EMBO I. 5: 1071-1076.

Harley, C.B., J. Lawrie, M. Betlach, H.W. Boyer, and J. Hedgpeth. 1988. Transcription initiation at the tet promoter and effect of mutations. Nucleic Acids Res. 16: 7269-7285.

Harley, C.B., J. Lawrie, H.W. Boyer, and J. Hedgpeth. 1990. Reiterative copying by $E$. coli RNA polymerase during transcription initiation at mutant pBR322 tet promoters. $\mathrm{Nu}$ cleic Acids Res. 18: 547-552.

Heyden, B., C. Nüsslein, and H. Schaller. 1975. Initiation of transcription within an RNA-polymerase binding site. Eur. J. Biochem. 55: 147-155.

Hsu, M.-T., J.D. Parvin, S. Gupta, M. Krystal, and P. Palese. 1987. Genomic RNAs of Influenza viruses are held in a circular conformation in virions and in infected cells by a terminal panhandle. Proc. Natl. Acad. Sci. 84: 8140-8144.

Hunt, D.M., E.F. Smith, and D.W. Buckley. 1984. Aberrant polyadenylation by a Vesicular Stomatitis virus mutant is due to an altered L protein. I. Virol. 52: 515-521.

Iverson, L.E. and J.K. Rose. 1981. Localized attenuation and discontinuous synthesis during vesicular stomatitis virus transcription. Cell 23: 477-484.

Jacks, T., H.D. Madhani, F.R. Masiarz, and H.E. Varmus. 1988. Signals for ribosomal frameshifting in the Rous sarcoma virus gag-pol region. Cell 55: 447-458.

Jacques, J.-P. and M.S. Susskind. 1990. Pseudotemplated transcription by $E$. coli RNA polymerase at a mutant promoter. Genes \& Dev. 4: 1801-1810.

Kassavetis, G.A., P.G. Zentner, and E.P. Geiduschek. 1986. Transcription at bacteriophage T4 variant late promoters. $J$. Biol. Chem. 261: 14256-14265.

Kolakofsky, D. and L. Roux. 1987. The molecular biology of paramyxoviruses. In The molecular basis of viral replication (ed. P. Bercoff), pp. 277-297. Plenum Publishing, New York.

Kunkel, T.A. and A. Soni. 1988. Mutagenesis by transient misalignment. J. Biol. Chem. 263: 14784-14789.

Liao, S.-M., T.H. Wu, C.H. Chiang, M.M. Susskind, and W.R. McClure. 1987. Control of gene expression in bacteriophage P22 by a small antisense RNA. I. Characterization in vitro of the Psar promoter and the sar RNA transcript. Genes \& Dev. 1: 197-203.

McGeoch, D.J. 1979. Structure of the gene N: Gene NS intercistronic junction in the genome of Vesicular Stomatitis virus. Cell 17: 673-681.

Machida, C., Y. Machida, and E. Ohtsubo. 1984. Both inverted repeat sequences located at the ends of ISl provide promoter functions. J. Mol. Biol. 177: 247-267.

Martin, C.T., D.K. Muller, and J.E. Coleman. 1988. Processivity in early stages of transcription by T7 RNA polymerase. Biochemistry 27: 3966-3974.

Moss, B. 1990. Replication of poxviruses. In Virology (ed. B.N. Fields), pp. 685-703. Raven Press, New York.

Nüsslein, C. and H. Schaller. 1975. Stabilization of promoter complexes with a single ribonucleoside triphosphate. Eur. J. Biochem. 56: 563-569.

Patel, D.D. and D.J. Pickup. 1987. Messenger RNAs of a strongly expressed late gene of cowpox virus contain 5'-terminal poly(A) sequences. EMBO /. 6: 3787-3794.

Paterson, R.G. and R.A. Lamb. 1990. RNA-editing by G-nucleotide insertion in Mumps virus P-gene mRNA transcripts. $J$. Virol. 64: 4137-4145.

Pelet, T., J. Curran, and D. Kolakofsky. 1991. The $P$ gene of
Bovine Parainfluenza Virus 3 expresses all three reading frames from a single mRNA editing site. EMBO $J$. 10: 443448.

Robertson, J.S., J.S. Schubert, and R.A. Lazzarini. 1981. Polyadenylation sites for influence virus mRNA. I. Virol. 38: 157163.

Rose, J.K. 1980. Complete intergenic and flanking gene sequences from the genome of vesicular stomatitis virus. Cell 19: $415-421$

Rose, J.K., H.F. Lodish, and M.L. Brock. 1977. Giant heterogeneous polyadenylic acid on vesicular stomatitis virus mRNA synthesized in vitro in the presence of S-adenosylhomocysteine. I. Virol. 34: 550-559.

Schubert, M., J.D. Keene, R.C. Herman, and R.A. Lazzarini. 1980. Site on the Vesicular Stomatitis Virus genome specifying polyadenylation and the end of the L gene mRNA. $J$. Virol. 34: 550-559.

Schwer, B. and H.G. Stunnenberg. 1988. Vaccinia virus late transcripts generated in vitro have a poly(A) head. $E M B O J$. 7: 1183-1190.

Schwer, B., P. Visca, J.C. Vos, and H.G. Stunnenberg. 1987. Discontinuous transcription or RNA processing of vaccinia virus late messengers results in a $5^{\prime}$ poly|A) leader. Cell 50: 163-169.

Shippen-Lentz, D. and E.H. Blackburn. 1990. Functional evidence for an RNA template in telomerase. Science 247: 546552.

Simpson, L. and J. Shaw. 1989. RNA editing and the mitochondrial cryptogenes of kinetoplastid protozoa. Cell 57:355366.

Takeuchi, K., K. Tanabayashi, M. Hishiyama, Y.K. Yamada, A. Yamada, and A. Sugiura. 1990. Detection and characterization of Mumps virus V protein. Virology 178: 247-253.

Thomas, S.M., R.A. Lamb, and R.G. Paterson. 1988. Two mRNAs that differ by two nontemplated nucleotides encode the amino coterminal proteins $\mathrm{P}$ and $\mathrm{V}$ of the paramyxovirus SV5. Cell 54: 891-902.

Vidal, S., J. Curran, and D. Kolakofsky. 1990a. Editing of the Sendai virus $\mathrm{P} / \mathrm{C}$ mRNA by $\mathrm{G}$ insertion occurs during mRNA synthesis via a virus-encoded activity. I. Virology 64: 239-246.

1990b. A stuttering model for Paramyxovirus P mRNA editing. EMBO J. 9: 2017-2022.

Wagner, L.A., R.B. Weiss, R. Driscoll, D.S. Dunn, and R.F. Gesteland. 1990. Transcriptional slippage occurs during elongation at runs of adenine or thymine in E. coli. Nucleic Acids Res. 18: 3529-3535.

Weir, J.P. and B. Moss. 1984. Regulation of expression and nucleotide sequence of a late Vaccinia Virus gene. I. Virol. 51: 662-669.

Wright, C.F. and B. Moss. 1989. Identification of factors specific for transcription of the late class of Vaccinia Virus genes. I. Virol. 63: 4224-4233.

Wu, T.-H., S.M. Liao, W.R. McClure, and M.M. Susskind. 1987. Control of gene expression in bacteriophage $P 22$ by a small antisense RNA. II. Characterization of mutants defective in repression. Genes \& Dev. 1: 204-212.

Yager, T.D. and P.H. von Hippel. 1987. Transcript elongation and termination in Escherichia coli. In Escherichia coli and Salmonella typhimurium (ed. F.C. Neidhardt, J.L. Ingraham, K.B. Low, B. Magasanik, and H.E. Umbarger), pp. 1231-1240. American Society for Microbiology, Washington, D.C.

Yu, G.L., J.D. Bradley, L.D. Attardi, and E.K. Blackburn. 1990. In vivo alteration of telomere sequences and senescence caused by mutated Tetrahymena telomerase RNAs. Nature 344: 126-132. 


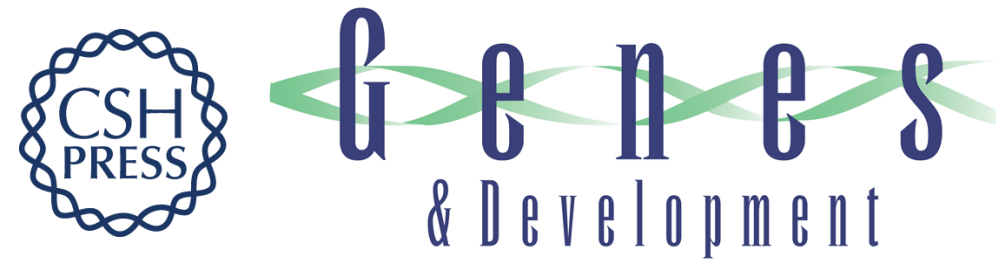

\section{Pseudo-templated transcription in prokaryotic and eukaryotic organisms.}

$J \mathrm{P}$ Jacques and D Kolakofsky

Genes Dev. 1991, 5:

Access the most recent version at doi:10.1101/gad.5.5.707

References This article cites 51 articles, 16 of which can be accessed free at:

http://genesdev.cshlp.org/content/5/5/707.full.html\#ref-list-1

License

Email Alerting Receive free email alerts when new articles cite this article - sign up in the box at the top Service right corner of the article or click here.

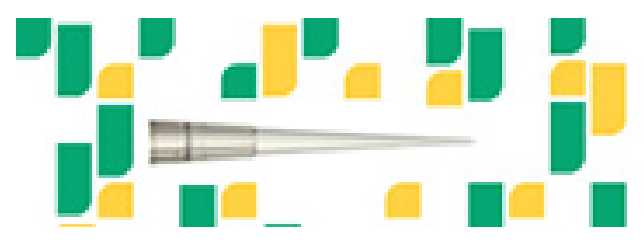

Focused on your science.

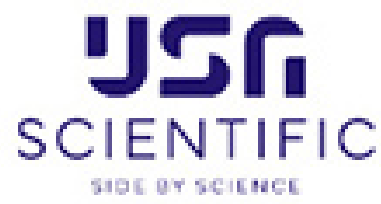

Copyright (c) Cold Spring Harbor Laboratory Press 